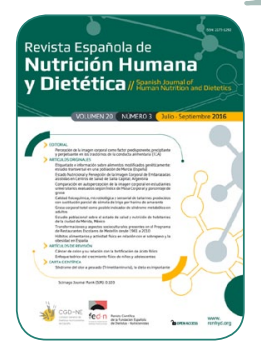

ORIGINAL

\title{
Transformaciones y aspectos socioculturales presentes en el Programa de Restaurantes Escolares de Medellín desde 1961 a 2010
}

\author{
Luz Marina Arboleda Montoya ${ }^{a, *}$ \\ a \\ Universidad de Antioquia, Colombia. \\ *marina.arboleda@udea.edu.co
}

Recibido el 2 de marzo de 2016; aceptado el 18 de julio de 2016.

\section{PALABRAS CLAVE}

Asistencia alimentaria;

Cultura;

Percepción;

Planificación de menús;

Política nutricional;

Colombia.
Transformaciones y aspectos socioculturales presentes en el Programa de Restaurantes Escolares de Medellín desde 1961 a 2010

\section{RESUMEN}

Introducción: La alimentación es un acto sociocultural complejo, que contempla dimensiones biológicas, nutricionales, políticas y sociales, y su imbricación hace que se presenten inequidades, como las situaciones de escasez y accesos a la alimentación por medio de programas sociales. El objetivo de este trabajo es identificar las transformaciones del programa de restaurantes escolares de Medellín (Colombia) e interpretar la percepción que tienen los beneficiarios de este programa.

Material y Métodos: Perspectivas cualitativa e histórica; etnografía e historia del tiempo presente. La etnografía se realizó con escolares beneficiarios y sus madres y padres, concejales, coordinadores del programa, manipuladoras y nutricionistas. Para recolectar la información histórica, se revisaron informes y correspondencia del Archivo Histórico de Medellín y actas del Archivo del Concejo Municipal de Medellín.

Resultados: El programa ha tenido diferentes modelos en la prestación del servicio y diferente cobertura; ha conservado la simpatía de los ediles y funcionarios de las diferentes secretarías. Este se configuró en los inicios, 1935, desde una visión de caridad, instituido por mujeres de la élite social, luego pasó por una visión higienista, nutricional, de derecho a la alimentación y por último, de seguridad alimentaria y nutricional. En los discursos y en las preparaciones suministradas se identifica la ausencia de referentes socioculturales.

Conclusiones: Los programas de restaurantes escolares contribuyen a disminuir el sufrimiento generado por el hambre en escolares, pero no siempre son diseñados pensando en los aspectos culturales que comporta la alimentación. 
Processing and sociocultural issues in the School Restaurants Program in Medellin from 1961 to 2010

\section{KEYWORDS}

Food Assistance;

Culture;

Perception;

Menu Planning;

Nutrition Policy;

Colombia

\section{ABSTRACT}

Introduction: Diet is a complex sociocultural act providing biological, nutritional, political and social dimensions. Interlocking them creates inequalities such as food shortages and access to food through social programs. The aim of this study is to identify the changes in the program of school restaurants in Medellin (Colombia), and to interpret the perception of the program's beneficiaries.

Material and Methods: Qualitative and historical perspectives; ethnography and history of the present time. Ethnography was conducted with school beneficiaries and their parents, councilors, program coordinators, food handlers and nutritionists. Historical Archives of Medellin and Minutes Archive Medellin Municipal Council were reviewed to collect the historical information, reports and correspondence.

Results: The program has different models of service provision and different coverage; it has retained the sympathy of the mayors and officials of the various secretariats. This was set up at the beginning, in 1935, from a perspective of charity school by elite women, and then it had a hygienist and nutritional vision of right to food; and finally, a food and nutrition safety vision. In the supplied speeches and preparations, the absence of cultural references is identified.

Conclusions: School Restaurants programs help to reduce the suffering caused by hunger, but they are not always designed thinking about the cultural aspects that involves food.

\section{CITA}

Arboleda Montoya LM. Transformaciones y aspectos socioculturales presentes en el Programa de Restaurantes Escolares de Medellín desde 1961 a 2010. Rev Esp Nutr Hum Diet. 2016; 20(3): 216 - 223. doi: 10.14306/ renhyd.20.3.218

\section{INTRODUCCIÓN}

Resolver el problema alimentario ha sido una prioridad de la humanidad entera durante toda su existencia. Pueblos enteros han adaptado su forma de vida en torno a la obtención del sustento alimentario para sobrevivir ${ }^{1}$, y para lograrlo, los grupos sociales han identificado y clasificado los alimentos y preparaciones según les proporcionen placer, saciedad o salud ${ }^{2}$; estableciéndose con ello sistemas alimentarios prácticos e implícitos que les indica lo que se deben comer, con quién, cuándo, dónde y para qué; e instaurándose una dinámica social alimentaria diferenciada para cada grupo poblacional. No obstante, dichas dinámicas, no siempre han garantizado el acceso a los alimentos ni la satisfacción de las necesidades alimentarias de los sujetos, presentándose así situaciones de hambre.

Tanto las catástrofes naturales como la acción del hombre, que con guerras fratricidas, el uso desmedido de la industrialización que destruye el ecosistema y la implementación de políticas económicas y alimentarias, han impedido que los grupos más desprotegidos de la sociedad accedan a los alimentos; ocasionando subnutrición y hambre, además de la llamada "hambre oculta", que se presenta por la baja e inadecuada ingesta de micronutrientes, conllevando a problemas nutricionales y de salud, y, en muchas ocasiones, a la sensación de abandono por parte de la sociedad.

Para afrontar dichas situaciones, las sociedades han establecido diversas estrategias que involucran tanto a los entes estatales como a la sociedad civil, las cuales han traspasado las fronteras de los países hasta volverse parte del orden mundial. Prueba de ello es la creación de la Organización de las Naciones Unidas para la Agricultura y la Alimentación -FAO- en 1943, como ente coordinador de los esfuerzos para resolver la problemática alimentaria a nivel mundial ${ }^{3}$.

Al igual que la mayoría de los países pobres, Colombia ha venido ejecutando intervenciones para mitigar el hambre mediante la instauración de programas y proyectos 
alimentarios a nivel local; no obstante, todo este esfuerzo será en vano mientras no se adopten políticas tendientes a garantizar el derecho a la alimentación adecuada y culturalmente aceptada.

Una de las ciudades que más programas ha implementado es Medellín, dada su agravada situación alimentaria por presentar el mayor índice de inequidad en el país ${ }^{4}$, por el continuo flujo de desplazados que recibe como producto del conflicto armado y por el aumento de la violencia en las comunas más populares, con las llamadas fronteras invisibles que causan desplazamiento de poblaciones a su interior ${ }^{5}$.

A pesar de la cuantiosa inversión y los esfuerzos realizados por la sociedad y el Estado local para mejorar la precaria situación alimentaria y nutricional de la población, estas situaciones persisten. Los indicadores de desnutrición e inseguridad alimentaria en la población en general así lo demuestran: el 32,6\% de la población menor de 18 años presenta baja talla para la edad, y el $9 \%$ presenta bajo peso para la talla. La percepción de inseguridad alimentaria en los hogares de la ciudad es alta, pues más de la mitad $(55,6 \%)$ se perciben en inseguridad alimentaria 6 , y el $13,4 \%$ presentan bajo consumo de calorías y de nutrientes ${ }^{7}$. A lo anterior se le suma la gran cantidad de población ubicada en los estratos bajo-bajo, y bajo-medio $(10,6 \%$ y $29,9 \%$ respectivamente) $)^{8}$.

Debido a lo anterior, es cada vez más corriente encontrar personas recorriendo las calles hurgando en las canecas (cubos) de basura, con la esperanza de hallar alimentos que puedan llevar a sus hogares; mujeres resignadas haciendo largas filas para recibir un paquete de alimentos, que acaso alcance para una semana; recursivas madres tasando para sus hijos la última ración disponible de alimentos, a sabiendas que otra vez, ellas, se acostaran sin comer; y los niños y niñas que asisten a la escuela con el aliciente de recibir, quizás, la única comida que consumirán en el día, tal como lo hacen los más de 57.000 .000 de niños beneficiarios de programas de Restaurantes Escolares en América Latina ${ }^{9}$

En esta ciudad, históricamente la población en que más se ha intervenido, sin lugar a dudas, es la escolar. Este grupo ha sido especialmente cobijado por el Programa de Restaurantes Escolares, el cual ha sido manejado en los últimos cincuenta años por la municipalidad y mantenido su vigencia como uno de los programas más importantes de asistencia social en el ámbito local.

En esta investigación se le da gran relevancia a dicho programa y especialmente a categorías socioculturales como el gusto, las preferencias y los rechazos, las formas de preparar y servir los alimentos, y el comensalismo que se da en el momento del consumo alimentario, por considerarse que deben ser tenidas en cuenta en el diseño de programas de complementación para garantizar que este acto se haga en condiciones de dignidad, aporte los nutrientes requeridos y responda a los aspectos simbólicos y a los significados que los sujetos le atribuyen a este acto ${ }^{10}$. Además, se articula al mandato de la Observación General 12 de la Comisión de los Derechos Sociales, Económicos y Culturales ${ }^{11}$, en la cual se dice que para que la alimentación se considere adecuada debe ser culturalmente aceptada.

El período comprendido en esta investigación abarca desde 1961, año en que se institucionalizó el programa por parte del Concejo Municipal, hasta el año 2010. En esta se interpreta la visión tanto de quienes diseñan políticas y operan programas alimentarios, como de los beneficiarios, procurando dar respuestas a preguntas como: ¿cómo se ha transformado el programa de Restaurantes Escolares en Medellín entre 1961 y 2010?, ¿cuál es la percepción que tienen los beneficiarios del Programa de Restaurantes Escolares sobre el complemento alimentario y la alimentación en sus hogares?

Los objetivos del presente trabajo son, por un lado, identificar las transformaciones y los aspectos socioculturales presentes en el Programa de Restaurantes Escolares de Medellín de 1961 a 2010, y, al mismo tiempo, interpretar la percepción que tienen los beneficiarios del Programa de Restaurantes Escolares de Medellín sobre su alimentación y el complemento alimentario suministrado.

\section{MATERIAL Y MÉTODOS}

Población: En esta investigación participaron diferentes sujetos relacionados con el Programa de Restaurantes Escolares de Medellín, entre estos: Concejales, funcionarios de la secretaría de familia e inclusión social (antes Bienestar Social), nutricionistas, interventoras y manipuladoras; y también se tuvo en cuenta la otra cara de la moneda, es decir, los beneficiarios del programa de Restaurantes Escolares (Escolares beneficiarios y sus madres y padres).

Muestra: Los participantes de esta investigación fueron un total de treinta y siete actores del Programa de Restaurantes Escolares, con los cuales no se pretendió obtener una saturación de la información, sino lograr una muestra representativa tipológica, es decir, que participaran diferentes actores del Programa: trece madres de beneficiarios, dieciséis escolares, una nutricionista, una coordinadora, una manipuladora, dos exfuncionarias del programa, una interventora, y tres administradores públicos (dos funcionarios de la Secretaría de Bienestar Social y un Concejal). 
Metodología: Esta investigación se realizó con un enfoque de corte cualitativo que facilitó la comprensión de la realidad subjetiva que experimentan y significan los grupos poblacionales beneficiarios de programas alimentarios de Medellín. Asimismo, facilitó la comprensión de las dinámicas alimentarias, sistemas de clasificación y de significados que se configuran alrededor de la escasez alimentaria y del hambre, dinámicas que se establecen y surgen en las relaciones y acuerdos intrafamiliares ${ }^{12}$; permitió además, la comprensión e interpretación de la realidad como resultado de un proceso histórico de construcción a partir de las lógicas de los diversos actores sociales, haciendo énfasis en la valoración de lo subjetivo, lo vivencial y la interacción entre los sujetos de la investigación ${ }^{13}$.

El método utilizado fue el etnográfico, centrado en el estudio de grupos poblacionales que comparten características, intereses, formas de comprender y comportarse, estilos de vida, situaciones sociales y culturales; por lo tanto establecen y configuran significados que también son compartidos $^{13}$. En la investigación realizada, los beneficiarios de programas de complementación alimentaria comparten diariamente su cotidianidad, sus necesidades, la mesa, y los espacios que le dan sentido y significado a sus vidas. Específicamente en este estudio se siguieron los lineamientos de la etnografía enfocada, que se caracteriza por el establecimiento de objetivos en una temática señalada, con grupos poblacionales aislables en una sociedad y en áreas específicas de comportamiento o de creencias ${ }^{14}$.

De igual manera, se utilizó el enfoque histórico, que posibilita la comprensión de los fenómenos sociales y de las situaciones presentes, como producto de las interacciones y procesos del pasado. Dicha perspectiva se interesa en temas contemporáneos (como las políticas alimentarias) ${ }^{15}$ con el fin de comprender los factores que subyacen a dicho acontecimiento social, conllevando a la producción de sentido. Esta perspectiva se constituye en una dimensión relevante para comprender el proceso de instauración y cambio del Programa en cinco décadas. Específicamente, se utilizó la historia del tiempo presente, por ser una perspectiva de análisis de la contemporaneidad en su duración, que permitió identificar la duración y cambios del programa de restaurantes escolares con sus contingencias y azares ${ }^{16}$. Dichas perspectivas proporcionan elementos para comprender la problemática estudiada, la cual está inserta en la dinámica social y en procesos de larga de duración.

Técnicas utilizadas: Para la recolección de la información cualitativa se utilizaron tres técnicas: las entrevistas individuales, las entrevistas grupales y la observación participante; las cuales se aplicaron con la flexibilidad implícita en este enfoque y con el rigor propio de las investigaciones. La entrevista grupal, es una técnica de recolección de información muy utilizada en los campos de la salud y del merca- deo para indagar por problemáticas que afectan a grupos específicos de personas. En síntesis, se trata de reproducir el discurso ideológico cotidiano: creencias y expectativas, deseos, resistencias y temores conscientes e inconscientes, etc. ${ }^{17}$. Las entrevistas grupales realizadas a escolares, madres y padres giraron en torno a la identificación de gustos y prácticas alimentarias en sus hogares, y a la percepción y uso del complemento alimentario.

La entrevista individual cualitativa, permite profundizar y comprender la realidad investigada desde la perspectiva de los sujetos entrevistados ${ }^{18}$; en este estudio facilitó la comprensión del significado de la escasez alimentaria y del hambre desde las perspectivas de quienes experimentan esta realidad. Permitió, profundizar en la operatividad, funcionamiento, cambios y visión que tenían los coordinadores, nutricionistas, funcionarios públicos y concejales sobre los programas de restaurantes escolares.

La observación participante se utiliza para comprender y explicar las situaciones indagadas, identificando directamente los comportamientos, las motivaciones y motivos y las reacciones de los sujetos, permitiendo comprender las experiencias individuales y compartidas de los otros, convirtiendo al investigador en participe de las situaciones observadas $^{17}$. Esta técnica fue empleada en el momento en que los escolares consumían el complemento alimentario en las instituciones educativas.

En total se realizaron cinco entrevistas grupales y veinticuatro individuales, y tres observaciones participantes en el Programa.

Para realizar las técnicas se estructuraron tres guías de entrevista, una para los escolares, otra para sus padres y madres y otra para coordinadores, nutricionista, interventores y funcionarios públicos. Los guiones se utilizaron para orientar las conversaciones de acuerdo a los aspectos previstos, sin imponer el orden de la guía, para que estas se realizaran acorde a los requerimientos del entrevistado y del momento.

Proceso metodológico: El trabajo de campo de la investigación se realizó con madres y padres de familia de escolares y con escolares beneficiarios de dos instituciones educativas: Colegio Guayacanes Fundadores del barrio El Socorro, e Institución Educativa La Divisa, ubicada en el barrio que lleva su mismo nombre, en la comuna 13.

Para recolectar la información se revisó el Archivo Histórico de Medellín y el Archivo del Concejo Municipal de Medellín desde 1961 hasta el año 2010, revisando documentos, informes, actas y correspondencias que dieran cuenta del proceso de creación y cambio del Programa de Restaurantes Escolares (ver fichero adicional). Dicha información se revisó, identificando en cuál de las actas e informes, se debatían 
aspectos del Programa, luego se fichó, y con base en el contenido de este fichaje, se realizó el proceso de análisis e interpretación de los datos.

Sistematización y organización de la información: Finalizada la recolección de información, se dio inició a la organización de la misma. La información proveniente del Archivo Histórico de Medellín databa desde el año 1961 hasta el año 1992, que correspondían a actas del Concejo Municipal, e informes y comunicaciones de las Secretarías de Salud y de Educación; la del Archivo del Concejo Municipal de Medellín en físico de 1993 al 2003 y la información digital de 2004 en adelante, que contenían información proveniente de las actas de los debates del Concejo. Dicha información fue fichada en un archivo digital, identificando la fuente, el año de consulta, el número y año del acta, la cita, las palabras clave y las observaciones. La información proveniente de las entrevistas individuales y grupales, de beneficiarios y funcionarios públicos, se procedió a transcribir de forma literal, asegurando la calidad de dichas transcripciones para obtener la totalidad de la información, pues, en buena medida, la calidad del análisis depende de la calidad de la transcripción de los datos obtenidos.

Análisis de la información: La información proveniente de las actas e informes del Concejo Municipal y de las Secretarías de Salud y Bienestar Social, y de Educación, aportó una visión general del programa, permitiendo ubicar el contexto nacional e internacional en el que surgieron y se desarrollaron los programas de complementación alimentaria. El fichaje de la información archivística, contribuyó a identificar el proceso de cambio de los programas, permitiendo diferenciar la variación en los objetivos trazados, y los complementos brindados, entre otros. Asimismo, se seleccionaron los principales debates de los concejales y funcionarios de la Alcaldía, que contribuyeron a ampliar la comprensión de los cambios en la visión y funcionamiento del programa en la Ciudad.

La información proveniente de las entrevistas cualitativas individuales y grupales fue codificada y luego agrupada por temas, para lo cual se utilizó el programa de análisis de datos cualitativos, ATLAS.ti versión 6.7. El proceso anterior llevó a la progresiva inmersión de la información transcrita ${ }^{18}$ y a alcanzar un primer nivel de análisis de la información. Luego se estructuraron e interpretaron las categorías y se estableció una red de relaciones entre estas, permitiendo alcanzar la construcción de sentido y un mayor nivel de análisis. Dichas categorías fueron:

- Surgimiento, transformaciones y continuidades del Programa de Restaurantes Escolares.

- Situaciones de escasez alimentaria en los hogares y percepciones de los beneficiarios sobre el complemento alimentario.
Aspectos éticos: En esta investigación se tuvo en cuenta el relativismo cultural, el cual significa estudiar otras culturas en sus propios términos y evitar juicios de valor etnocéntricos sobre ellos. Los sujetos que participaron en la investigación lo hicieron de manera voluntaria y se respetó en todo momento su privacidad e intimidad, procurando que su bienestar físico y emocional no se viera afectado ${ }^{19}$. Antes de la participación se les hizo entrega y lectura del consentimiento informado, documento donde se consignó el objetivo y alcance de la investigación, se les pidió la autorización para grabar la conversación, explicándoles que dicho material sería utilizado sólo con fines académicos e investigativos. Se aseguró el anonimato de los participantes y la confidencialidad de la información durante el proceso de análisis de los datos y escritura de la información, evidenciándose en la sustitución de sus nombres por un código.

\section{RESULTADOS}

Surgimiento, transformaciones y continuidades del Programa de Restaurantes Escolares: El programa de restaurantes escolares comenzó su operación en 1935, con el nombre de Patronato Escolar bajo un esquema de beneficencia-caridad. Posteriormente, debido al crecimiento acelerado de la población de Medellín, se incrementó el número de pobres ${ }^{20}$ haciéndose más perentoria la construcción de escuelas públicas que albergaran a los hijos de estas familias, y las necesidades de restaurantes escolares para mejorar el rendimiento académico de los niños, ya que estos no podían aprender con hambre, se configuraron como un proceso civilizatorio y sostenido luego por el Estado. En estos comedores para niños pobres se servían comidas tradicionales, basadas en el concepto de "sopa escolar", enfatizando los esfuerzos en mantener a los niños saciados, con buen rendimientos escolar y disciplinados.

Las primeras consideraciones que se encuentran en las actas del Concejo, amén de las económicas, tuvieron que ver con aspectos higienistas, evidenciados en la importancia del lavado de manos, la limpieza de los escenarios y el adoctrinamiento de los menores; donde se pudo ver por primera vez cómo el modelo sugerido por la potencia económica fue acatado por el ejecutivo de la época y transformado en norma, sin tener en cuentas las adaptaciones necesarias que respetasen y mantuviesen los valores culturales propios.

Las preparaciones del Programa, en la década de los sesenta y setenta, se producían en un gran centro principal que planteaba problemas del orden de almacenaje de víveres, de manipulación de alimentos, de cocción a nivel industrial, y de transporte. De otra parte de la década de los setenta, datan los primeros rechazos alimentarios documentados del 
complemento recibido, relacionados con las horas inusuales para la ingesta, inadecuación de preparaciones para las horas de consumo y con las bajas temperaturas de las preparaciones suministradas. El modelo biomédico se instauró en el Programa con la creación del Instituto Colombiano de Bienestar Familiar (ICBF) y se difundió con el surgimiento de Escuelas y Facultades de Nutrición y Dietética. De nuevo el esquema fue implantado desde una política nacional, que a su vez fue eco de un discurso importado, que no tuvo en cuenta las particularidades de la cultura donde era ejecutado. Desde esta óptica, la alimentación fue concebida desde la dimensión de la nutrición, como la formulación de una medicalización de nutrientes que debió ser suministrada para la recuperación de los menores con deficiencias nutricionales, que aunque fue una dimensión fundamental, debió estar articulada con dimensiones simbólicas y culturales.

En los noventa se sumó al discurso biomédico, la perspectiva del derecho a la alimentación, la cual fue incorporada en los programas de complementación alimentaria del municipio, como derecho ya consagrado en la nueva constitución política de $1991^{21}$ y en los pactos internacionales a favor de la infancia. En los debates del Concejo de Medellín, se hacía alusión a los derechos de la niñez y adolescencia, a la participación comunitaria y a la corresponsabilidad de la familia respecto a la crianza de sus hijos. En la práctica dicha participación se limitó sólo a la ayuda que prestaban las madres, de manera gratuita en la elaboración del complemento alimentario; sin embargo, nunca se les tuvo en cuenta, ni a ellas, ni a los escolares, ni a la comunidad en el diseño y evaluación del programa. En los primeros años del nuevo milenio, la política social se instauró bajo el enfoque de la Seguridad Alimentaria y Nutricional, que contempló los ejes de disponibilidad, acceso, consumo, aprovechamiento biológico y calidad e inocuidad de los alimentos; enfoque que aportó, en teoría, una visión y estrategias amplias e integrales, pero que en su implementación, en alguna medida, se limitó a la entrega de un complemento alimentario y a la educación nutricional.

Así mismo, la política social viró cada vez más hacia la atención a la primera infancia, enmarcada en la perspectiva mundial de la Inversión y Capital Social22. Como trasfondo de esta política internacional se creó en la Ciudad el Programa de Buen Comienzo, dirigido a madres gestantes y a niños lactantes. De esta manera, se configuró una política de protección a la infancia, que deja desprotegida al resto de la población, y que sólo piensa en el futuro. Políticas focalizadas en los menores, que separaron a los miembros de las familias en el acto alimentario y generaron inequidades a su interior.

Situaciones de escasez alimentaria en los hogares de beneficiarios de Restaurantes Escolares y percepciones sobre el complemento alimentario: Las madres de los escolares percibieron el Programa como una estrategia para acceder a alimentos: vieron el complemento alimentario adecuado para asegurar el crecimiento y desarrollo de sus hijos; consideraron que era una estrategia muy adecuada para evitar la inasistencia de estos a las escuelas porque cuando se presentaba escasez de alimentos en los hogares pudieron mandarlos a la institución educativa con la tranquilidad de que allí obtendrían alimentos; y vieron también un modo de optimizar el dinero porque, como no requirieron el refrigerio, lo utilizaron para acceder a alimentos de mejor calidad. Aunque otras madres consideraron que el complemento suministrado a sus hijos no siempre fue agradable al gusto, ni se les brindó en la cantidad suficiente para saciarles el hambre. A los escolares por su parte les agradó el complemento, aunque dijeron que a veces lo que no les agradaba eran las combinaciones de los alimentos suministrados. Por ejemplo, les ofrecían pan, chocolate y piña o jugo con pan o arepa (torta de pan de maíz); esta última composición la rechazaban porque no tenían forma de remojar el pan/arepa, o porque se los ofrecían a horas de la mañana en las que estaban acostumbrados a una bebida caliente. También extrañaban el comensalismo y las formas acostumbradas de consumo de los alimentos en sus hogares.

De otro lado, las familias de los beneficiarios subsistían en situación de escasez de alimentos permanente, lo que conllevó a la presencia de hambre y hambre oculta. Fueron las mujeres las que llevaban el mayor peso de esta problemática y quienes implementaban las estrategias para enfrentarla, las cuales fueron variadas e instauradas como rebusque (economía informal, por ej. vender dulces en los semáforos); las principales fueron: la mendicidad, el recorrido (tránsito callejero para conseguir alimentos, o bien desechados para su venta aunque en buen estado, o bien a través de de centros de abastecimiento gratuito para personas necesitadas) y la adscripción a programas de complementación alimentaria. La escasez también influyó en las decisiones de compra de alimentos, que se orientaron hacia aquellos que fueran favorables, saciadores, afines a sus costumbres y del gusto de la familia ${ }^{23}$. Los alimentos que cumplían estas características fueron: el arroz, las lentejas, los espaguetis, la panela (azúcar moreno o sin refinar) y la arepa; los cuales, por su gran contenido de carbohidratos y grasas, tuvieron repercusiones en la calidad de las preparaciones que consumían, y en la formación del gusto de la familia, lo que configuró formas propias de alimentación que, en muchas ocasiones, conllevaban al hambre oculta. No obstante, estas mujeres manifestaron sentirse resignadas y agradecidas por los alimentos a los que tenían acceso, porque, según ellas, al menos tenían algo que comer y con qué calmar el hambre, sobre todo la de los niños. La falta de alimentos en el hogar se hizo más insoportable para ellas porque advertían que sus hijos no comprendían dicha situación. 
Las mujeres fueron también las que tuvieron las mayores responsabilidades en la compra, elaboración y el consumo de alimentos en los hogares. Además, fueron quienes más sufrieron las consecuencias de la escasez de alimentos, porque siempre eran quienes consumían lo que quedaba, cuando quedaba, después de haber brindado comida a sus hijos $\mathrm{y}$ al resto de la familia.

De hecho, cuando el dinero era aún más escaso, o alguno de los productos que les eran indispensables subía de precio, ellas establecían estrategias para acceder a los alimentos y realizaban ajustes para mantener el consumo acostumbrado. Otras de estas estrategias implementadas consistían en hacer sólo dos comidas en el día, desayuno y comida o desayuno y merienda, y acostarse más temprano y levantarse más tarde. Éstas fueron también las que establecían las redes de solidaridad cuando el acceso a los alimentos comenzaba a dificultarse.

\section{DISCUSIÓN}

Si se tiene en cuenta lo que afirma Calero ${ }^{24}$, la escuela pública no considera la dimensión intercultural y estética en las prácticas de alimentación, ya que su principio fundamental es saciar el hambre y disciplinar. Se podría pensar que el principio que rige a los programas como el de restaurantes escolares es sólo mejorar el estado nutricional de los escolares y evitar su deserción; para lo cual se configura la idea de la alimentación sólo desde referentes biológicos, y se dejan de lado las dimensiones socioculturales de la alimentación; que por demás no serían necesarias si la pretensión es saciar el hambre. Pero si el principio del programa se configurara desde el derecho a la alimentación, que integra aspectos de adecuación y referentes culturales, sería mandatorio comprender cómo se da el acto alimentario en la cotidianidad de los sujetos beneficiarios y de la sociedad, para integrar dicha dinámica en el diseño y ejecución del programa.

Por consiguiente, la efectividad de un programa depende de la armonía que exista entre múltiples factores que deben sopesarse cuando se planea su instauración. Los aspectos políticos determinan la voluntad y el compromiso que se tendrá con el programa. Los económicos fijan su cobertura y su duración. Los aspectos técnicos garantizan la operatividad, la calidad y la inocuidad de los alimentos que se suministran. La participación de profesionales en nutrición respalda el consumo balanceado y el aprovechamiento de los alimentos. $Y$ los aspectos socioculturales aseguran la aceptación que un grupo poblacional hará del complemento alimentario ofrecido. Está sincronía aún no se ha logrado, en parte, por la deuda en lo relacionado con los aspectos socioculturales de la alimentación, aun ausentes en los programas. Por lo tanto, al elaborar programas de complementación alimentaria, es indispensable identificar aspectos relacionados con la alimentación de los beneficiarios tales como sus gustos, rechazos, formas de preparar los alimentos, formas de servir, ambiente en el que se consumen y los sentidos y significados atribuidos a los diferentes momentos de la alimentación. Por ejemplo, al elaborar menús, tomar como base las preparaciones que gustan en los hogares de los beneficiarios, brindar combinaciones alimentarias adecuadas e incluso opciones de elección alimentarias. Al servir el complemento alimentario, propiciar ambientes familiares, festivos y sociales, que involucren la lúdica, el gusto y el comensalismo, y se integren los docentes; ambientes donde se ofrezcan alimentos a la vez nutritivos y gustosos, acordes a las costumbres locales.

De esta manera, los programas de complementación alimentaria se instaurarían sobre los fundamentos de la ética, justicia social y derecho a la alimentación adecuada, suficiente y culturalmente aceptada; y se haría de estos un acto humano y estético, no un programa para pobres o enfermos en el cual sería mucho más relevante la simple pregunta de "¿cómo les pareció la comida?" a quienes se beneficiaron de esta, que la realización de elaborados estudios técnicos. Lo anterior, conllevaría a un cambio de paradigma que posibilitaría la comprensión de las interacciones y dinámicas alimentarias, y con este, un cambio de preguntas, donde ya no sólo se indagaría por el "¿qué se consume?", sino que ya se harían indispensables preguntas como: ¿con quién se lleva a cabo el acto alimentario?, ¿cuándo se da el acto alimentario?, ¿qué representa la alimentación?, o ¿qué significa el acto alimentario?

\section{CONCLUSIONES}

En principio las políticas eran de orden local, preocupadas exclusivamente por mantener económicamente el programa, y aumentar cobertura para así mejorar el rendimiento académico de los menores y disminuir su deserción escolar. Luego se adoptaron recomendaciones del modelo higienista, y más adelante, con la entrada del ICBF, dichas políticas se adscribieron a los lineamientos del orden nacional, preocupándose en mayor medida, por instaurar un modelo biomédico en el cual primasen los aspectos técnico-nutricionales, a las que luego se adicionaron las perspectivas de derecho a la alimentación y seguridad alimentaria y nutricional. Los programas de restaurantes escolares contribuyen a disminuir el sufrimiento generado por el hambre en escolares; no obstante, no siempre son diseñados pensando en los aspectos culturales que comporta la alimentación de los beneficiarios como las formas de preparación, las combinaciones de las preparaciones, las preparaciones suministradas acorde a la hora y clima, el gusto alimentario y las preferencias, entre otros. 


\section{FICHEROS ADICIONALES}

\section{Archivos consultados:}

- Archivo Histórico de Medellín

- Archivo Concejo Municipal de Medellín

- Concejo Municipal de Medellín

http://renhyd.org/index.php/renhyd/article/downloadSuppFile/218/1649

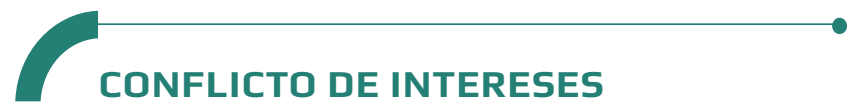

La autora expresa que no hay conflictos de interés al redactar el manuscrito.

\section{REFERENCIAS}

(1) Rotberg RI, Rabb TK. El hambre en la historia: el impacto de los cambios en la producción de alimentos y los modelos de consumo sobre la sociedad. Madrid, España: Siglo Veintiuno de España; 1990.

(2) Aguirre P. Seguridad alimentaria: una visión desde la antropología. En: Sabulsky J, Ezpeleta ML, Chesta M, Fundación CLACYD, editores. Enfoque integral del desarrollo en la infancia: el futuro comprometido: ponencias y debates. Córdoba, Argentina: Fundación CLACYD; 2004.

(3) Organización de las Naciones Unidas para la Agricultura y la Alimentación. El estado de la inseguridad alimentaria en el mundo 2009 - Crisis económicas: repercusiones y enseñanzas extraídas. Roma, Italia: FAO; 2009.

(4) Álvarez MC, Benjumea MV, Roldán P, Maya ML, Martinez MM, Montoya EC, et al. Perfil alimentario y nutricional de los hogares urbanos de Medellin. Medellín, Colombia: Gobernacion de Antioquia; 2005.

(5) Naranjo G, editor. Seguimiento y balance sobre el desplazamiento forzado, la población afectada y las políticas públicas: Medellín 2004-2007. Medellín, Colombia: Instituto de Estudios Políticos - Universidad de Antioquía; 2007. (Movilidad, Migraciones y Desplazamiento Forzado).

(6) Alcaldía de Medellín. Proyecto de Restaurantes Escolares para la infancia y la adolescencia y Vaso de Leche Escolar. Alcaldía de Medellín; 2010.

(7) Arboleda LM, Ochoa AM, Isaza UA. Prácticas, acceso y cambios alimentarios en Medellín [Internet]. Medellín, Colombia: Secretaria de Bienestar Social; 2010 [citado 27 de septiembre de 2016]. Disponible en: http://www.libreroonline.com/ libros/45109/arboleda-montoya-Iuz-marina-ochoa-hoyos- ana-maria-alberto-isaza-uber/practicas-acceso-y-cambiosalimentarios-en-medellin.html

(8) Departamento Administrativo de Planeación. Vivienda. En: Encuesta de Calidad de Vida 2008. Medellín, Colombia: Alcaldía de Medellín; 2009. p. 79-118.

(9) da Silva JG, Ortega J, Faiguenbaum S. Estrategias de desarrollo, políticas públicas y seguridad alimentaria en América Latina y el Caribe. Santiago, Chile: Rimisp; 2008.

(10) Gracia M. Antropología de la alimentación. En: Prat Carós J, Martínez A, editores. Ensayos de antropología cultural: Homenaje a Claudio Esteva-Fabregat. Barcelona: Ariel Antropología; 1996.

(11) Naciones Unidas. Observación general № 12 (1999) - EI derecho a una alimentación adecuada (artículo 11 del Pacto). En: Comité de Derechos Económicos, Sociales y Culturales Informe sobre los períodos de sesiones 20 Y 21․ Nueva York y Ginebra: Naciones Unidas; 2000. p. 98-106.

(12) Souza MC, Ferreira S, Cruz O, Gomes R. Investigacion social: teoria, metodo y creatividad. Argentina: Lugar Editorial; 2003.

(13) Galeano ME. Estrategias de investigación social cualitativa: el giro de la mirada. Medellín: La Carreta; 2004.

(14) Boyle JS. 9 - Estilos de etnografía. En: Morse JM, editor. Asuntos críticos en los métodos de investigación cualitativa. Medellín, Colombia: Universidad de Antioquia; 2003. p. 188-213.

(15) Le Goff J. Pensar la Historia: modernidad, presente y progreso. 1 a ed. Barcelona: Paidós Ibérica; 1997.

(16) Fazio H. La historia del tiempo presente: una historia en construcción. Historia crítica. 1998; (17): 47-57.

(17) Sandoval CA. Investigación cualitativa. Bogotá, Colombia: ARFO Editores e Impresores; 2002.

(18) Amezcua M. La entrevista en grupo. Características, tipos y utilidades en investigación cualitativa. Enfermería Clínica. 2003; 13(2): 112-7.

(19) Bonilla-Castro E, Rodríguez P. Métodos cuantitativos y cualitativos. En: Más allá del dilema de los métodos - La investigación en ciencias sociales. 3a ed. Bogotá, Colombia: Grupo Editorial Norma; 2005. p. 77-103.

(20) Álvarez V. Poblamiento y población en el valle de Aburrá y Medellín 1541-1951. En: Melo JO, editor. Historia de Medellín. Bogotá, Colombia: Compañía suramericana de seguros; 1996. p. $57-84$

(21) Colombia, Presidencia de la República. Constitución política de Colombia, 1991. Bogotá, Colombia: Presidencia de la República; 1991.

(22) Inza A, Urteaga E. La inversión social en educación. Revista Mexicana de Ciencias Políticas y Sociales. 2012; 57(216): 10318.

(23) Aguirre P. Aspectos socioantropológicos de la obesidad en la pobreza. En: La obesidad en la pobreza: un nuevo reto para la salud pública. Washington, DC.: Organización Panamericana de la Salud; 2000. p. 13-26.

(24) Calero S. Pedagogías sociales y alimentación escolar. En: Actas del I Encuentro Latinoamericano de Investigadores sobre Cuerpos y Corporalidades en las Culturas. 1a ed. Rosario, Argentina: Investigaciones en Artes Escénicas y Performáticas; 2012. 